\title{
Dynamically formed poly (vinyl alcohol) ultrafiltration membranes with good anti-fouling characteristics
}

\author{
Li Na*, Liu Zhongzhou, Xu Shuguang \\ Polymer Membrane Division, Research Center for Eco-Environmental Sciences, Chinese Academy of Sciences, \\ P.O. Box 2871, Beijing, 100085, PR China
}

Received 9 July 1999; received in revised form 21 September 1999; accepted 8 October 1999

\begin{abstract}
The anti-fouling poly (vinyl alcohol) TFC membranes were dynamically prepared when an aqueous solution containing poly (vinyl alcohol), cross-linking agents and additives passed through porous substrate membranes under definite pressure followed by heat treatment resulting in cross-linking reaction and drying. By means of dead-end filtration the poly (vinyl alcohol) solution deposited on the surface and entered the pores of porous substrate and thus an even poly (vinyl alcohol) gel layer was formed on both the external and internal surface. The effects of poly (vinyl alcohol) concentration, dynamic coating time, additive concentration, cross-linking agent concentration, curing time and support membrane on pure water flux and protein rejection of the resultant membranes were studied. Membrane morphology was characterized by SEM studies. The modified membranes were investigated in ultrafiltration experiments with pepsin to assess their resistance to fouling. The experimental results prove that modified membranes with an even poly (vinyl alcohol) hydrogel layer show dramatically high anti-fouling characteristics compared to inadequately modified and unmodified membranes. Moreover, it is possible that a series of dynamic membranes within broader molecular weight cut-off can be readily obtained by suitable control and combination of various preparative conditions. (C2000 Elsevier Science B.V. All rights reserved.
\end{abstract}

Keywords: Ultrafiltration; Anti-fouling; Dynamic membrane; Poly (vinyl alcohol); Hydrogel layer

\section{Introduction}

Today, more and more attention in membrane separation technology has been focused upon solutions to decrease and eliminate irreversible membrane fouling because of its serious hindrance to the development and application of membrane science and technology. Much important research work is on the technique of surface modification which is based on the principle that increasing hydrophilicity of membrane surface

\footnotetext{
* Corresponding author. Fax: +86-0106-2923441.

E-mail address: shgxu@mail.rcees.ac.cn (L. Na).
}

can generally improve the ability to resist fouling by inhibiting non-specific binding between the membrane surface and retained molecules, particularly proteins.

Poly (vinyl alcohol) (PVA) polymer, with highly hydrophilic character, good film-forming properties and outstanding physical and chemical stability, is a kind of excellent membrane material for preparation of a hydrophilic membrane. In fact, considerable work has been carried out in the area of PVA-RO composite membrane, where PVA or PVA co-polymer was prepared as a selective skin layer of composite RO membrane. The PVA skin layer provides the resulting membranes with high water permeation rate, good 
anti-fouling nature, excellent integrity in acidic and alkaline environments and remarkable resistance to abrasion.

For example, Petersen [1] reviewed the published activity over the period 1961-1993 on RO membranes made with PVA. Immelman et al. [2] prepared both flat-sheet and tubular composite RO membranes by depositing aqueous solutions of PVA and potassium peroxydisulphate on asymmetric poly (arylether sulphone) [PES] substrate membranes. Lang et al. [3,4] prepared PVA composite membranes for RO with various cross-linking agents including aldehydes, dialdehydes and malic acid. The anti-fouling low-pressure RO membranes-LF10 series commercialized by Nitto Denko Corporation in 1997 indicated the direction in the development of the RO membrane. Their membranes were formed by coating a traditionally made polyamide support membrane with a PVA layer that not only eliminates the negative charge of the support membrane but also provides hydrophilicity and chlorine resistance, thereby largely improving the anti-fouling characteristics. Besides this, PVA is also widely used as the material of selective skin of composite pervaporation membranes.

The studies on preparation of PVA membranes applied to UF are relatively rare compared to that of PVA membranes for reverse osmosis or pervaporation. Since the UF process is usually used in separation and concentration of foods, pharmaceuticals and biological substances, especially for the concentration of dilute protein solutions and separation of proteins with various molecular weights, it requires more exploitation of the use of PVA in UF membranes to reduce adsorption of proteins or other macromolecules concerned on the membrane surface. Few reported works have thus far been carried out on PVA-UF membranes. $\mathrm{Li}$ and Barbari [5] inferred that the use of cross-linked hydrogel for the modification of UF membranes was a large unexplored area. Hydrogels provide smooth, hydrophilic surfaces with minimal protein-binding and can be manipulated easily in terms of thickness and the degree of cross-linking to fit desired UF needs. $\mathrm{Li}$ and Barbari prepared composite UF membranes by spin-coating cross-linked PVA hydrogel onto regenerated cellulose UF membranes that provided high anti-fouling to bovine serum albumin (BSA) as indicated by nearly $100 \%$ pure water flux recovery. They considered that since the PVA hydrogel surface exhibited minimal protein adsorption, any irreversible fouling that manifested itself in less than $100 \%$ pure water recovery was associated with the surface of regenerated cellulose support. This was consistent with the study of Fane et al. [6] who inferred that although the adsorption was largely on the top surface and the amount of protein adsorbed within the pores could be a minor fraction of the total quantity adsorbed, it could have a profound effect on membrane performance. Nabeet et al. [7] proved by their experiments that aggregation in the pores or on the pore surface could be an important mechanism of flux decline and that the chemistry of the substrate membrane surface directly affected the flux decline during the ultrafiltration of protein solution.

So, if the hydrophilicity of the internal surface of membrane is increased as well as that of the external surface through suitable surface treatment, it is expected that the resultant elimination of macrosolute adsorption within the pores and of blockage of the passage should lead to markedly improved resistance to fouling and increase in separation efficiency for macromolecular mixtures and membrane permeation stability. However, most composite membranes have hydrophobic support and much attention has been focused on the hydrophilicity and formation of the ultra-thin skin layer of the composite membranes but not on the hydrophilicity and internal pore fouling of the support membrane.

The present study focuses on the development of good anti-fouling PVA-based TFC membrane for UF. The PVA composite membranes were formed when an aqueous solution containing PVA, cross-linking agents and additives passed through porous substrate membranes under pressure. By means of this dynamic coating method the PVA casting solution deposited on the surface and entered the pores of the porous substrate by pressure and deposited on the internal surface of the support. Then the deposit was submitted to heat treatment leading to cross-linking reactions and drying. Thus an even cross-linked PVA ultra-thin layer formed on the external and internal surface. Both UF and MF membranes have been used as porous substrates. In order to obtain satisfactory flux, macroporous substrates with high pure water flux and high porosity are preferable. By ultrafiltration experiments with protein feed solution, the 
Table 1

The support membranes characteristics

\begin{tabular}{llllr}
\hline Membrane designation & Material & Total thickness ${ }^{\mathrm{a}}(\mu \mathrm{m})$ & Nominal MWCO $^{\mathrm{b}}($ Dalton$) /$ pore diameter $(\mu \mathrm{m})$ & Water flux $^{\mathrm{c}}\left(\mathrm{l} / \mathrm{m}^{2} \mathrm{~h}\right)$ \\
\hline PA-1 & PAN & 190 & 60,000 & $460 \pm 20$ \\
PV-1 & PVDF & 165 & 150,000 & $800 \pm 20$ \\
PV-2 & PVDF & 150 & 500,000 & $1800 \pm 50$ \\
NL-1 & Nylon & 155 & $1 \mu \mathrm{m}$ & $2200 \pm 100^{\mathrm{d}}$ \\
\hline
\end{tabular}

${ }^{a}$ Including the non-woven support of thickness approx. $112 \mu \mathrm{m}$.

${ }^{\mathrm{b}}$ Based on above $90 \%$ rejections of proteins.

${ }^{c}$ The test conditions are $0.1 \mathrm{MPa}$, room temperature.

$\mathrm{d}$ The test conditions are $0.05 \mathrm{MPa}$, room temperature.

membranes were characterized with respect to their resistance to fouling and their 'cleanability'.

\section{Experimental}

\subsection{Materials}

Fully hydrolyzed PVA powder having a degree of polymerization of 1799 was obtained from Beijing Hongxing Chemical Industry Factory. Fully hydrolyzed PVA powder having average molecular weight of 11,000-31,000 was obtained from Aldrich. Glutaraldehyde (GA), the cross-linking agent, was obtained as a $50 \mathrm{wt} \%$ GA aqueous solution. The glutaraldehyde concentration in this paper all refers to the concentration of $50 \mathrm{wt} \%$ GA. Methanol, acetic acid and sulfuric acid were of reagent grade and were used without further purification. The water was de-ionized.

The flat-sheet microfiltration membranes and ultrafiltration membranes used as the support membranes were made by traditional phase-inversion method on polyester non-woven fabric in our laboratory and workshop. In principle, almost all porous polymeric membranes had high water flux and permitted permeation of PVA. The support membranes used in this study are listed in Table 1. Prior to use, the support membranes were soaked in distilled water to remove the glycerol-wetting agent and the support membrane was tested to determine the pure water flux and then strictly selected to ensure that its water flux was within the given range for its type as indicated in Table 1. A discussion of casting conditions of support membranes falls beyond the scope of this paper. The support membranes were pre-treated by being immersed in a $60 \mathrm{vol} \%$ aqueous solution of alcohol for $24 \mathrm{~h}$ to increase its hydrophilicity and then coated according to the following procedures.

\subsection{Apparatus and modification procedures}

The dynamic coating of support membranes and protein fouling experiments were carried out by using a $50 \mathrm{ml}$ stirred dead-ended filtration cell with $10 \mathrm{~cm}$ membrane area. A flow sheet of the experimental apparatus is shown in Fig. 1.

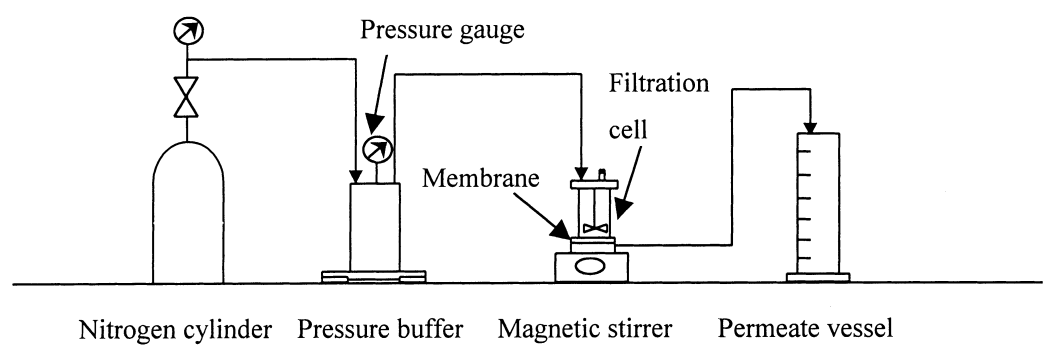

Fig. 1. Flow sheet of the experimental apparatus. 
Aqueous PVA solution prepared by dissolving the polymer in distilled water completely and then being filtered was added with additive, glutaraldehyde as cross-linking agent, methanol as quencher, acetic acid as buffer, sulfuric acid as catalyst. The mixture was stirred until homogeneous and heated to $45^{\circ} \mathrm{C}$. Then the $50 \mathrm{~m} 1$ mixture was quickly poured into the stirred cell in which a support membrane had been loaded, and was pressured through the membrane by using a nitrogen cylinder. During a pre-determined period of filtration, the dynamic membrane was formed. The membrane was taken out and cross-linked in an oven at $50^{\circ} \mathrm{C}$ for a pre-determined period. Finally, the membrane was swollen in distilled water and stored for performance experiments. The particular preparation and testing conditions are indicated in the figures and tables in Section 3.

\subsection{Membrane performance test}

\subsubsection{Permeability and retention studies}

In order to study the effects of various preparative conditions on the performance of the resultant composite membranes, three primary parameters were determined: pure water flux $\left(1 / \mathrm{m}^{2} \mathrm{~h}\right)$, protein aqueous solution flux $\left(1 / \mathrm{m}^{2} \mathrm{~h}\right)$ and the protein rejection $\left(R_{\mathrm{e}}\right)$ which is defined as $R_{\mathrm{e}}=1-C_{\mathrm{p}} / C_{\mathrm{b}}$, where $C$ is the concentration of protein in the permeation and $C_{\mathrm{b}}$ is the concentration of protein in the bulk solution. The protein rejection and molecular weight cut-off (MWCO), defined as above $90 \%$ rejection of proteins, were measured using two kinds of proteins: pepsin and bovine serum albumin (BSA). The molecular weight and isoelectric points (PI) of these proteins are listed in Table 2. Flux data were measured at room temperature under pre-determined operating pressure using the apparatus of Fig. 1. Protein rejection values were obtained using UV at $280 \mathrm{~nm}$ (Model UV-120-02, Shimadzu).

Table 2

Molecular weights and isoelectric points of the proteins used

\begin{tabular}{lll}
\hline Protein & Molecular weight & Isoelectric point \\
\hline Pepsin & 35,000 & Below 1.0 \\
BSA & 65,000 & 4.8 \\
\hline
\end{tabular}

\subsubsection{Fouling study methods}

The fouling experiments were consecutively carried out with the apparatus of Fig. 1:

1. The pure water fluxes were measured volumetrically until the fluxes remained constant for three successive readings (over $5 \mathrm{~min}$ ).

2. The UF experiment with an aqueous solution of protein was performed until the fluxes remained constant for three successive readings (over $5 \mathrm{~min}$ ). The nominal molecular weight of the chosen protein is smaller than the MWCO of the tested composite membranes so as to indicate the internal fouling situation of the tested membranes. Pepsin aqueous solution was used to assess the resistance of membranes of 60,000 (MWCO) to fouling.

3. The membrane was taken out and briefly rinsed with de-ionized water for approximately $5 \mathrm{~min}$. and the pure water fluxes were measured again until the flux remained constant for three successive readings (over $5 \mathrm{~min}$ ).

4. The same way as Step (2).

5. The same way as Step (3).

\subsubsection{SEM studies}

A number of scanning electron micrographs was recorded to compare the morphology of the modified membranes with that of the unmodified membranes. All samples were soaked in $50 \%$ glycerol aqueous solution for at least $10 \mathrm{~h}$, dried in vacuum, fractured in liquid nitrogen and gold-coated. Fig. 2 (a, b) shows the top surface and cross-section of NL-1 support membrane magnified $\times 2000$, respectively. Fig. 3(a, b) shows the top surface and cross-section of a modified membrane magnified $\times 2000$, respectively. Fig. 4(a, b) shows the top surface of another modified membrane magnified $\times 10000$ and cross-section magnified $\times 2000$.

\section{Results and discussions}

\subsection{Effects of various preparative conditions on membrane performance}

The permeability and structures of PVA-TFC membrane were much different from that of the substrate 


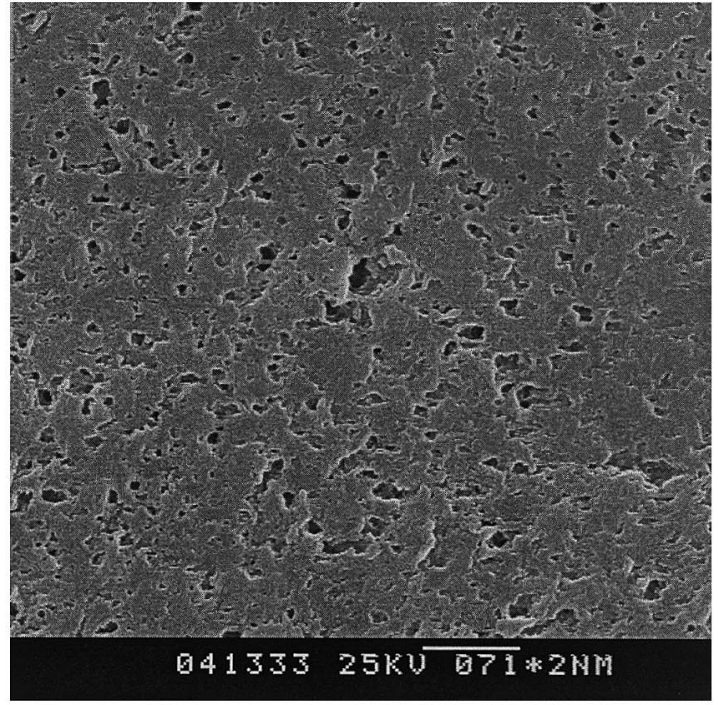

(a)

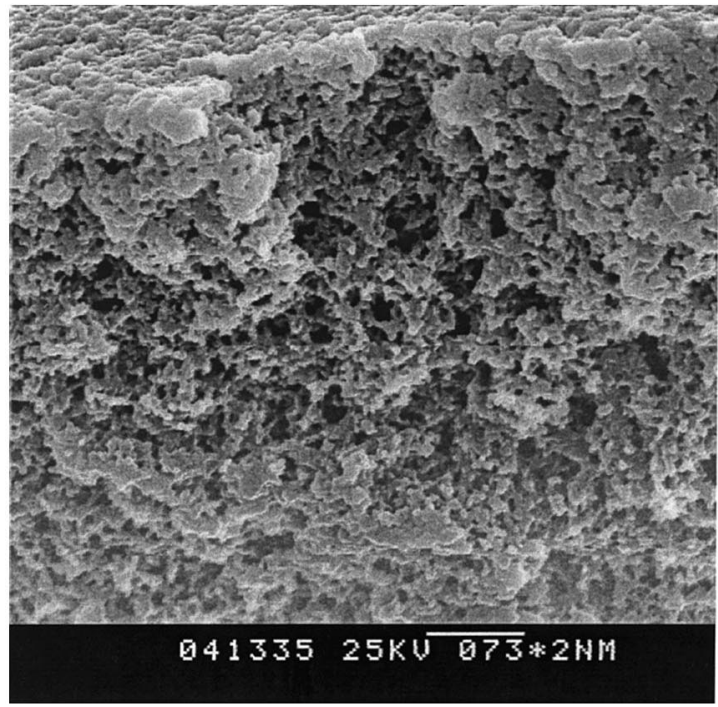

(b)

Fig. 2. (a) Scanning electron micrograph of the top surface of NL-1 support membrane magnified $\times 2000$. (b). Scanning electron micrograph of the cross-section of NL-1 support membrane magnified $\times 2000$.

membrane since a PVA cross-linked layer on both the external and the internal surface covered by the substrate membrane. Various preparative conditions used in the process can cause more or less effects on the performance of the resultant membrane and should be studied in detail.

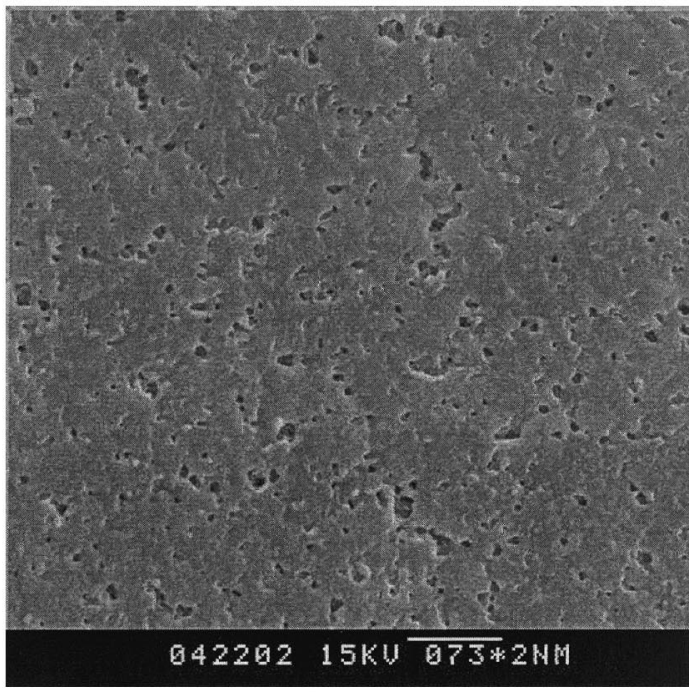

(a)

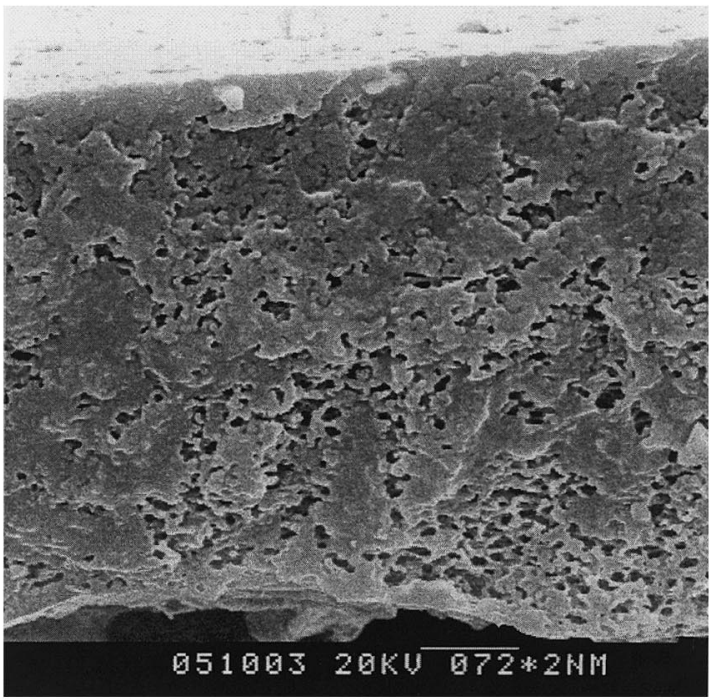

(b)

Fig. 3. (a) Scanning electron micrograph of the top surface of the PVA composite membrane magnified $\times 2000$. (b) Scanning electron micrograph of the cross section of the PVA composite membrane magnified $\times 2000$.

\subsubsection{Effect of PVA concentration}

The effect of PVA concentration in the casting solution on membrane performance was studied using PA-2 membrane as substrate, which was dynamically coated with coating solutions containing from 0.3 to $1 \mathrm{wt} \%$ PVA whereas other preparative conditions were 


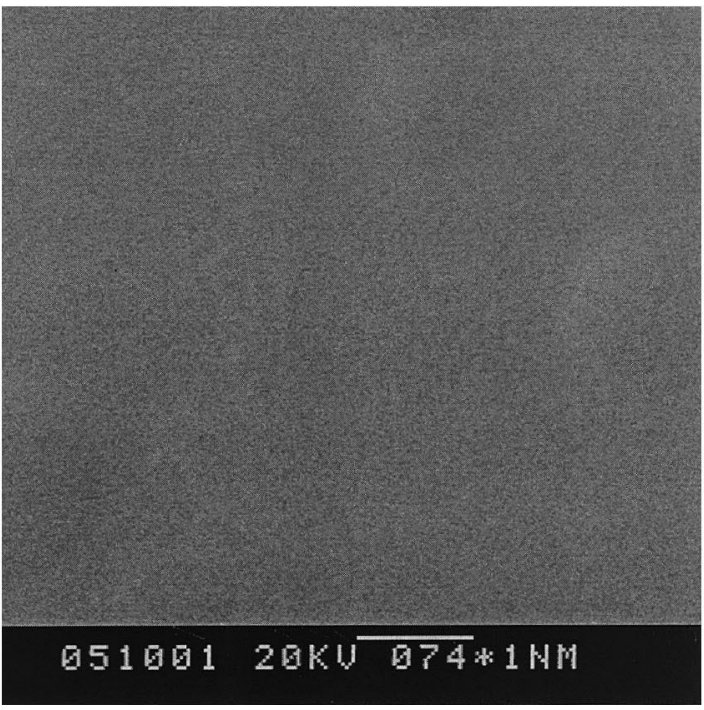

(b)

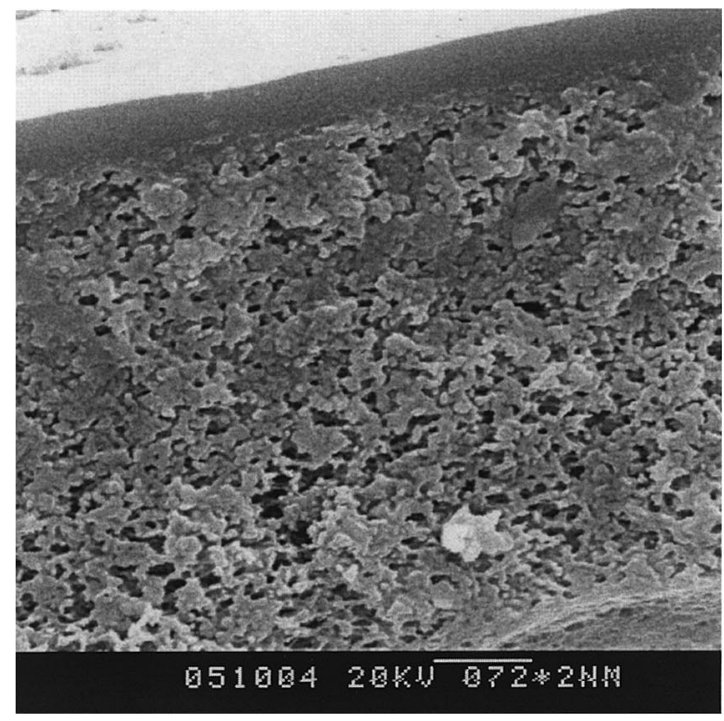

(a)

Fig. 4. (a) Scanning electron micrograph of the top surface of the PVA composite membrane magnified $\times 10,000$. (b) Scanning electron micrograph of the cross-section of the PVA composite membrane magnified $\times 2000$.

fixed. The UF experimental data illustrated in Fig. 5 show that an increase in PVA concentration resulted in a decrease in pure water flux and an increase in BSA retention. The increase of PVA concentration means that more PVA penetrates into and through the membrane during formation procedures. Under the pressure
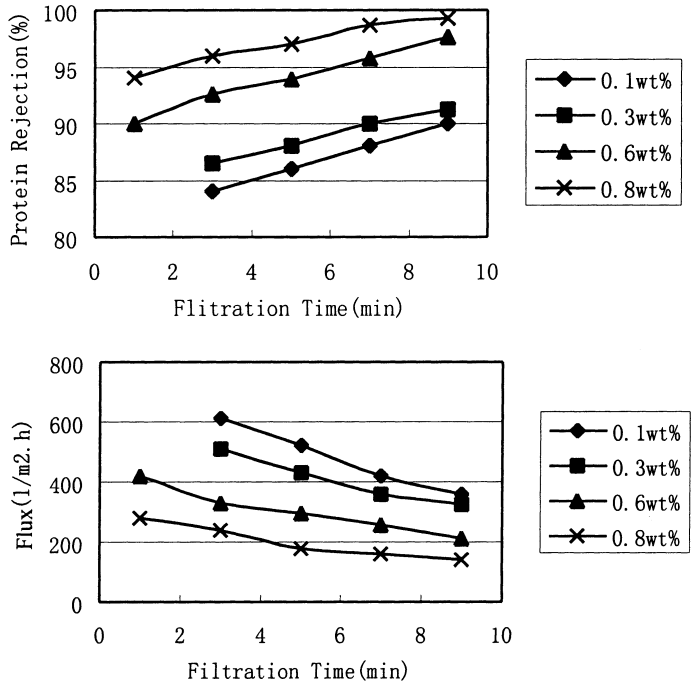

Fig. 5. Effect of PVA concentration and dynamic coating time on the performance of PVA-1799 coated PV-2 membrane. Conditions of membrane preparation: concentration of glutaraldehyde, $0.3(\mathrm{vol} \%)$; volume concentration of methanol, acetic acid and sulfuric acid, $0.2,0.3,0.1 \%$; the additive concentration, 12 vol\%; operating pressure, $0.2 \mathrm{MPa}$; curing time: $7 \mathrm{~h}$. Conditions of UF testing: 0.1 MPa, room temperature.

one part of PVA passes through the pores and leaves the membrane while other part of PVA stays on both, the external and internal surface of the membrane as a boundary layer. An increase in concentration provides more PVA into the boundary layer and becomes cross-linked. This leads to the result of smaller pore diameters, which is responsible for the reduction in water flux and the increase in protein retention.

\subsubsection{Effect of dynamic coating time}

In the preparation process the dynamic coating time of PVA aqueous solution through support membrane can have an evident effect on membrane performance. To study the effect, the coating time was varied from 1 to $13 \mathrm{~min}$ under pressure of $0.2 \mathrm{MPa}$. The results in Fig. 5 show that BSA retention increased whereas pure water flux decreased with increasing coating time. During the formation procedure one part of PVA passes through the substrate membrane and leaves the membrane under pressure and other part of PVA stays on both the external and internal surface and forms a dynamic layer on both the external and internal surface. It is expected that when filtration time increases, 

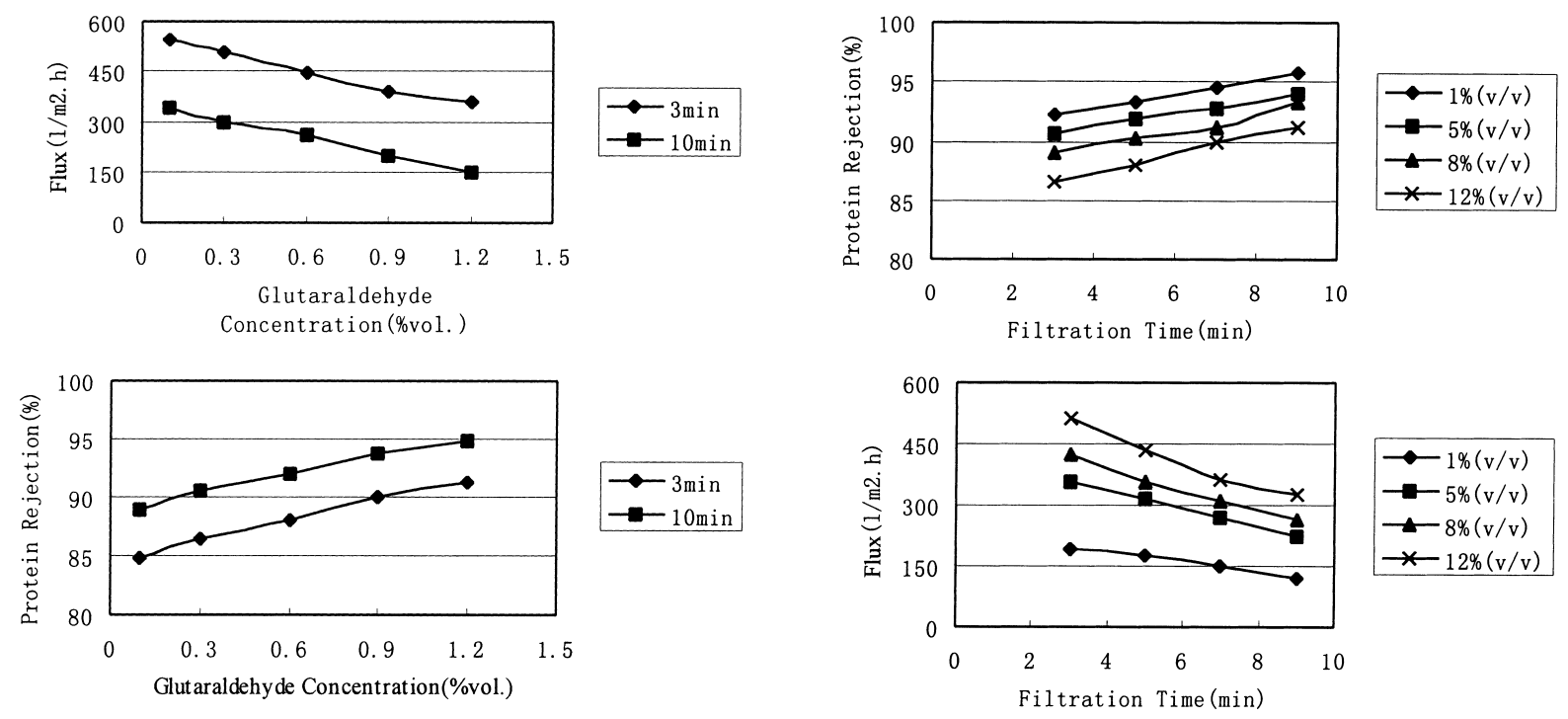

Fig. 6. Effect of glutaraldehyde concentration in PVA casting solution on the performance of PVA-1799 coated PV-2 membrane. Conditions of membrane preparation: PVA-1799 concentration, $0.3 \mathrm{wt} \%$; the additive concentration, $12 \mathrm{vol} \%$; volume concentration of methanol, acetic acid and sulfuric acid, 0.02, 0.03, $0.01 \%$; operating pressure, $0.2 \mathrm{MPa}$; curing time, $5 \mathrm{~h}$. Conditions of UF testing: $0.1 \mathrm{MPa}$, room temperature.

more PVA deposits on the coating layer. The smaller pores on the substrate membrane would be affected initially and be filled quickly and the dynamic layer on the top surface of the substrate membrane will even cover the smaller pores with increasing the filtration time. In the meanwhile, the bigger pores are not covered completely with the dynamic layer, but their actual pore diameters decrease because the coating layer formed on the wall of the pores is thicker with the increasing of filtration time. All of these cause a decrease in the water flux and a lower molecular weight cut-off (MWCO).

\subsubsection{Effect of cross-linking agent concentration}

Fig. 6 illustrates the effect of glutaraldehyde concentration in PVA casting solution on the performance of the composite membrane. The glutaraldehyde concentrations were varied from 0.1 to 1.2 vol\% and the other parameters were fixed. Increasing glutaraldehyde concentration led to increasing BSA retention and decreasing pure water flux of PVA composite membrane. It is unclear whether cross-linking results

Fig. 7. Effect of additive concentration on the performance of PVA-1799 coated PV-2 membrane. Conditions of membrane preparation: PVA-1799 concentration in casting solution, $0.3 \mathrm{wt} \%$; glutaraldehyde concentration, $0.3 \mathrm{vol} \%$; the volume concentration of methanol, acetic acid, sulfuric acid, 0.02, 0.03, $0.01 \%$; operating pressure, $0.2 \mathrm{MPa}$; curing time, $7 \mathrm{~h}$. Conditions of UF testing: $0.1 \mathrm{MPa}$, room temperature.

in more coating or a denser coating. There are some reports on the structure of PVA cross-link including the effect of cross-linking agent on the structure of PVA hydrogel, such as the study of Burczak et al. [8]. They illustrated that the cross-linked PVA hydrogel consists of networks of macromolecular chains (macromolecular mesh). Their experimental results demonstrated that the increase in the concentration of the added cross-linking agent formed the denser cross-linked PVA hydrogel network. According to their study, it is expected that the addition of GA results in denser coating at the same PVA concentration and the density will increase with the increase of GA concentration, which results in increase in BSA and decrease in pure water flux.

\subsubsection{Effect of additive concentration}

The effect of additive concentration on membrane performance is shown in Fig. 7. The additive used was PEG-400. An increase in additive concentration appreciably increased pure water flux of composite membrane, but slightly decreased BSA retention. PEG-400 
Table 3

Effect of curing time on the performance of composite membrane ${ }^{\mathrm{a}}$

\begin{tabular}{lccc}
\hline Curing time & 1 hour & 4 hours & 7 hours \\
\hline PWP $\left(1 / \mathrm{m}^{2} \mathrm{~h}\right)$ & 330.6 & 310.2 & 290.4 \\
$\operatorname{Re}(\mathrm{BSA})(\%)$ & 90.7 & 92.3 & 94.1 \\
\hline
\end{tabular}

${ }^{a}$ Conditions of membrane preparation: PVA concentration, $0.6 \%$; glutaraldehyde concentration, $0.3 \%$ vol.; the volume concentration of methanol, acetic acid, sulfuric acid, 0.02, 0.03, 0.0 $1 \%$; dynamic coating time, $5 \mathrm{~min}$; operation pressure, $0.2 \mathrm{MPa}$. Conditions of UF testing: $0.1 \mathrm{MPa}$, room temperature.

is a pore-forming agent here. When PVA composite membrane was soaked in distilled water during the formation procedures, PEG was separated out from the PVA membrane and dissolved into the distilled water. So increasing PEG concentration results in forming more pores and this may give a chance to form large pores, thereby leading to an increase in pure water flux and the slight decrease in protein retention.

\subsubsection{Effect of curing time}

The effect of curing time on the performance of the membrane is shown in Table 3. The longer curing time resulted in higher BSA retention, but lower flux. This may be because curing induced water removal from the gel structure and is responsible for a closer position of PVA chains. However, the change is not very significant compared to those caused by the effects of the procedures shown in Sections 3.1.1-3.1.4. This may be related with the curing temperature, $45^{\circ} \mathrm{C}$, which was not very high.

\subsubsection{Effect of support membrane}

Since the pore wall of substrate membrane was covered with a cross-linked PVA layer and the resultant pore diameters were changed markedly, it is natural that the water flux of PVA-TFC membranes was much lower than those of their substrate membranes, and strongly affected by the performance of substrate membranes. The experimental results shown in Table 4 illustrated that the water flux of the composite membrane increased, whereas the protein rejection decreased with an increase in pore sizes of substrate membranes when all remaining variables were fixed at pre-determined values. It is quite evident that the effect of substrate on the flux and retention of the composite membrane was considerably large among
Table 4

Effect of support membrane on the performance of composite membrane ${ }^{a}$

\begin{tabular}{lcccr}
\hline Support membrane & PA-1 & PV-1 & PV-2 & NL-1 \\
\hline PWP (1/m ${ }^{2}$ h) & $<10$ & 100 & 190 & $1460^{\mathrm{b}}$ \\
$\operatorname{Re}($ BSA) $(\%)$ & - & 99.9 & 97.2 & - \\
\hline
\end{tabular}

${ }^{\text {a }}$ Conditions of membrane preparation: PVA-1799 concentration, $1 \mathrm{wt} \%$; glutaraldehyde concentration, $0.7 \mathrm{vol} \%$; the volume concentration of methanol, acetic acid, sulfuric acid, 0.04, 0.06, $0.02 \%$; dynamic coating time, $3 \mathrm{~min}$; operation pressure, $0.2 \mathrm{MPa}$. Conditions of UF testing: $0.1 \mathrm{MPa}$, room temperature.

$\mathrm{b}$ The test conditions are $0.05 \mathrm{MPa}$, room temperature.

the preparative conditions. Obviously the substrates with high water flux are preferable and it is possible to produce a PVA-TFC membrane with broader MWCO range by manipulating and controlling various preparative conditions when the substrate membranes with large pore diameter and high porosity are applied. Just considering the effect of substrate membrane, glutaraldehyde was chosen as a cross-linking agent because PVA cross-linking by glutaraldehyde can take place at room temperature and heat treatment at high temperature can be avoided which can cause the most significant flux loss as proved by Lang et al. [4]. Since the performance of some membranes is affected less by heat treatment at high temperature, such membranes can be chosen as substrates when the cross-linking agents must react with PVA at high temperature. So the process can use other kinds of the cross-linking agents without much loss of water flux of PVA composite membrane caused by heat treatment at high temperature.

\subsubsection{Effect of post-treatment}

During the preparation of PVA composite membranes, after the cross-linking reaction of PVA for a pre-determined period, the membrane was often soaked in distilled water to make the additive separated out and the PVA layer reach swelling equilibrium. Here, in order to investigate the stability of the PVA composite membrane in hot water, after the membrane was soaked in $30^{\circ} \mathrm{C}$ distilled water for 2 days, it was then soaked in $80^{\circ} \mathrm{C}$ distilled water for $24 \mathrm{~h}$ post-treatment. Table 5 shows the preparative conditions of two PVA composite membranes, $1^{\prime}$ and $2^{\prime}$. Test the pure water fluxes and protein retention of the two membranes with post-treatment 
Table 5

Conditions of membrane preparation to study the effect of post-treatment

\begin{tabular}{lll}
\hline Membrane designation & $1^{\prime}$ & $2^{\prime}$ \\
\hline Substrates & PV-1 & PV-2 \\
PVA-1799 concentration (wt\%) & 1 & 1 \\
Glutaraldehyde concentration (vol\%) & 0.6 & 0.6 \\
Concentration of methanol, acetic acid, sulfuric acid (vol\%) & $0.04,0.06,0.02$ & $0.04,0.06,0.02$ \\
Additive (PEG-400) Concentration (vol\%) & 12 & 12 \\
Dynamic coating time (min) & 5 & 5 \\
Operation pressure (MPa) & 0.2 & 0.2 \\
Curing time (h) & 7 & 7 \\
\hline
\end{tabular}

and the two membranes without post-treatment. The experimental results shown in Fig. 8 illustrate that the fluxes of PVA membranes with post-treatment are rather higher than that of the membranes without post-treatment while the difference of protein retention between them is not large. Protein retention
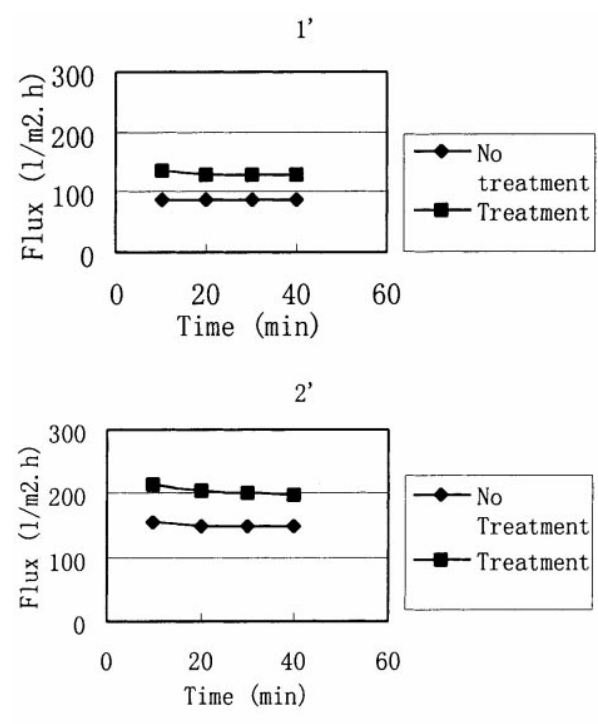

Protein Retention(\%)

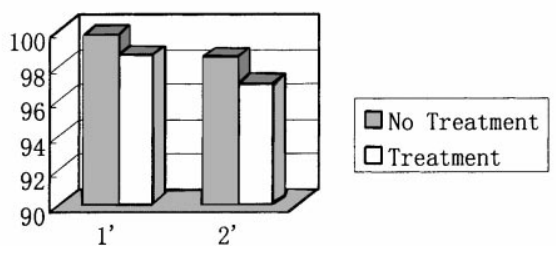

Fig. 8. Effect of post-treatment on the performance of PVA-1799-coated PV-1 and PV-2 membranes. of the membranes after post-treatment still remains rather high. This result may be related to the fact that the additive, PEG, is separated out more completely and some short-chain PVA molecules not cross-linked during cross-linking reaction, may dissolve into hot water during soaking. On the other hand, that the protein retention of the membranes is stable after treatment in $80^{\circ} \mathrm{C}$ hot water indicates the stability of the PVA composite membrane. The above results suggest that this post-treatment can improve the general performance of the PVA composite membrane. PVA is a kind of water-soluble polymer and the membrane prepared with PVA may dissolve in water during application processes if PVA does not proceed with the cross-linking reaction. The chemical cross-linking of PVA in an aqueous solution leads to the formation of an insoluble polymeric network. So it is expected that the cross-linked layer lasts longer than an uncross-linked layer. In the following work we will further study the stability of the PVA composite membrane in other media under certain conditions.

\subsection{Preparation of PVA composite membrane with different $M W C O$}

Figs. 2-4 show the differences of morphology among support membrane and its modified composite membranes. The support membrane was NL-1 membrane made in our lab. The preparatory conditions of modified membranes are shown in Table 6. It can be distinguished that the average pore size of modified membranes shown in Fig. 3(a) was not much smaller than that of support membrane shown in Fig. 2(a) and the pore size of modified membrane shown in 
Table 6

Preparative conditions of membranes of Figs. 3 and 4

\begin{tabular}{lll}
\hline Membrane & Membrane of Fig. 3 & Membrane of Fig. 4 \\
\hline Type & Modified membrane & Modified membrane \\
PVA-1799 concentration, (wt $\%)$ & 0.7 & 3 \\
PVA (M.W. 11,000-31,000) concentration (wt $)$ & 0.3 & 2 \\
Glutaraldehyde concentration, (vol\%) & 0.5 & 1 \\
Concentration of methanol, acetic acid, sulfuric acid (vol\%) & $0.04,0.06,0.02$ \\
Additive (PEG-6000) concentration (wt $\%)$ & 3 & $0.1,0.15,0.05$ \\
Dynamic coating time ( min) & 2 & 3 \\
Operation pressure (MPa) & 0.05 & 70 \\
Curing time (h) & 7 & 7 \\
\hline
\end{tabular}

Fig. 4(a) was so small that it could not be observed with SEM magnified $\times 10000$. This resulted from the difference in preparative conditions between the two modified membranes. So it is evident that PVA composite membranes with different diameters or MWCO can be prepared by suitable control of various preparative conditions and it is possible to dynamically make a series of PVA composite membranes within broad molecular weight cut-off.

Fig. 4 clearly shows a thick and smooth dynamic layer on the top surface while the coating thickness of the membrane in Fig. 3 is relatively small. From this it can be suggested that with the increase of filtration time or/and PVA concentration, the dynamic layer on the top surface increases quickly and creates a denser layer, and plays a much more important role in the performance of the PVA composite membrane. It can be expected that the combination of a very large pore size, low concentration and short filtration time produce the thinner PVA coating layer on the membrane surface, as shown in Fig. 3. The top dynamic layer is not a continuous layer so the pores can remain large.

The authors of this paper think that this dynamic formation method should not be limited to application just for preparing flat-sheet membranes for UF. It can be extended to the application for preparing composite membranes in other configurations in situ, such as tubular composite membranes and hollow fiber composite membranes. In fact, the authors have prepared hollow fiber PVA composite membranes with MWCO 35,000 in situ by this method and have proved that the composite membranes have good anti-fouling characteristics.

\subsection{Fouling studies}

Short-time fouling tests for the modified and unmodified membranes have been performed by the methods described in Section 2.3.2. The experimental results are shown in Figs. 9 and 10.

Fig. 9 shows typical flux versus time profiles for modified thin-gel composite membrane $3^{\prime}$ (MWCO, $60,000)$ and unmodified polysulfone membrane (MWCO, 60,000) that exhibit approximately the same

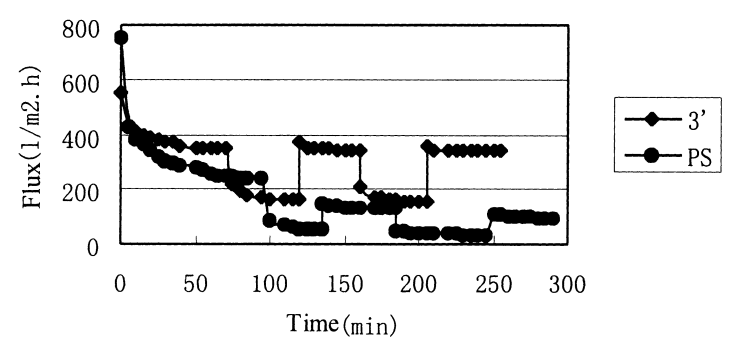

Fig. 9. Flux vs. time of modified membrane $3^{\prime}$ and unmodified polysulfone membrane that exhibit approximately same rejection of $0.1 \%$ pepsin aqueous solution.

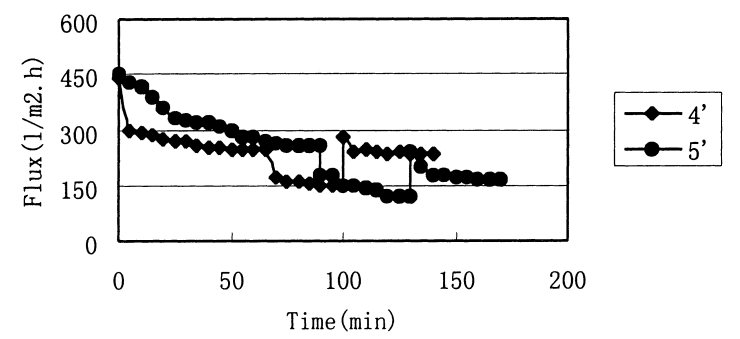

Fig. 10. Flux vs. time of two different modified membranes $4^{\prime}$ and $5^{\prime}$ that were dynamically coated for 3 and $1 \mathrm{~min}$, respectively. 
Table 7

Conditions of membrane preparation to study the anti-fouling characteristics

\begin{tabular}{|c|c|c|c|}
\hline Membrane designation & $3^{\prime}$ & $4^{\prime}$ & $5^{\prime}$ \\
\hline Substrates & PV-2 & $\mathrm{PV}-2$ & PV-2 \\
\hline PVA-1799 concentration (wt \%) & 0.5 & 0.8 & 0.8 \\
\hline Glutaraldehyde concentration (vol\%) & 0.2 & 0.3 & 0.3 \\
\hline Concentration of methanol, acetic acid, sulfuric acid (vol\%) & $0.02,0.03,0.01$ & $0.02,0.03,0.01$ & $0.02,0.03,0.01$ \\
\hline Additive (PEG-400) Concentration (vol\%) & 12 & 12 & 12 \\
\hline Dynamic coating time (min) & 3 & 3 & 1 \\
\hline Operation pressure (Mpa) & 0.2 & 0.2 & 0.2 \\
\hline Curing time $(\mathrm{h})$ & 6 & 6 & 6 \\
\hline
\end{tabular}

rejection of $0.1 \%$ pepsin solution, 71.7 and $70.7 \%$ respectively. The preparative conditions of the modified membrane $3^{\prime}$ are listed in Table 7. Fig. 9 shows that modified membrane $3^{\prime}$ exhibits stable pure water flux and protein solution flux during filtration and as good as $100 \%$ water flux recovery while the polysulfone membrane exhibits relatively rapid flux decline and low flux recovery. It shows that the modified membrane has excellent 'cleanability' and anti-fouling characteristics over a few cycles. Flux decline with time of suitably modified membranes appears to be controlled by the reversible formation of a protein layer adjacent to the hydrogel and compaction of membranes under pressure during filtration.

Fig. 10 shows typical flux versus time profiles for two different modified thin-gel composite membranes which exhibit 90.7 and $83.8 \%$ rejections of $0.1 \%$ BSA, respectively. The preparative conditions of the two membranes are listed in Table 7. The only difference between the preparation processes of the two membranes consists in dynamic coating time: 3 and $1 \mathrm{~min}$, respectively. From the above content, we can know that when other preparation conditions are fixed, increased coating time leads to the decrease of the flux. So the flux of Membrane $5^{\prime}$ should be higher than that of Membrane 4'. Fig. 10 shows that the flux of Membrane $5^{\prime}$ is higher than that of Membrane $4^{\prime}$ for the first $60 \mathrm{~min}$, but after $60 \mathrm{~min}$ the flux of Membrane $5^{\prime}$ is rather similar to that of the Membrane $4^{\prime}$. This indicates greater flux decline of Membrane $5^{\prime}$. As the difference of the coating times of the two membranes, 3 and $1 \mathrm{~min}$. respectively, is not very large, the difference of the fluxes between the two membranes appears to be not very large too, including at $150 \mathrm{~min}$ stage, according to Fig. 10. However, the degree of pure water flux recovery of Membrane $5^{\prime}$ is rather lower compared to that of Membrane $4^{\prime}$. This proves that Membrane $4^{\prime}$ is better than Membrane $5^{\prime}$ in anti-fouling characteristics. In addition, the membranes have been removed from service at different times because it took different time for the two membranes to reach stable flux and it appears to be easier for Membrane $4^{\prime}$ to reach stable flux than the Membrane 5'. From Fig. 10 we can distinguish the general decline situation of the two membranes. It can be proved that the more dynamic coating time, the more the stability of the flux and the greater degree of water flux recovery. The degree of pure water flux recovery of Membrane $4^{\prime}$ can reach as much as $100 \%$ according to experimental data while that of Membrane $5^{\prime}$ is low, which may result from the too short dynamic coating time. It is expected that when the dynamic coating time is too short, the PVA layer on the surface of substrate membrane is not uniform and some part of substrate membrane surface is exposed to feed solution which results in fouling and less 'cleanability'.

\section{Conclusion}

1. The anti-fouling PVA-TFC membranes can be dynamically prepared when an aqueous solution containing PVA, cross-linking agents and additives pass through porous substrate membranes under definite pressure followed by cross-linking reaction and drying.

2. The effects of preparative conditions on resulting composite membranes were investigated. Increasing PVA concentration of the casting solution, $\mathrm{dy}$ namic coating time, glutaraldehyde concentration and curing time, as well as decreasing additive concentration, all result in a decrease in flux and an increase in protein rejection. 
3. Due to the flexibility of the manipulation in the process, PVA composite membranes with different molecular weight cut-off can be readily obtained by suitable control of various preparative conditions and it is possible to dynamically make a series of PVA composite membranes with broad molecular weight cut-off.

4. On the basis of flux stability and pure water flux recovery tested with pure water and protein aqueous solution, modified membranes coated with an even PVA hydrogel layer show dramatically high anti-fouling characteristics and good 'cleanability' compared to inadequately modified membranes and unmodified membranes. The adequately modified membrane shows recoverable pure water flux and protein solution flux that makes them suitable for repeat usage applications especially for the concentration or separation of protein solutions.

\section{Acknowledgements}

This work was supported by the Chinese Academy of Sciences, Project KZ951-A1-201-02, KZ95T-05.

\section{References}

[1] R.J. Petersen, Composite RO and NF membrane, J. Membr. Sci. 83 (1993) 81-150.

[2] E. Immelman, D. Bezuidenhout, R.D. Sanderson, Poly (vinyl alcohol) gel sub-layers for reverse osmosis membranes. III. Insolubilization by cross-linking with potassium peroxydisulphate, Desalination 94 (1993) 115-132.

[3] K. Lang, T. Matsuura, G. Chowdhury, Preparation and testing of polyvinyl alcohol composite membranes for reverse osmosis, Can. J. Chem. Eng. 73 (1995) 686-692.

[4] K. Lang, S. Sourirajan, T. Matsura, G. Chowdhury, A study on the preparation of polyvinyl alcohol thin-film composite membranes and reverse osmosis testing, Desalination 104 (1996) 185-196.

[5] R.H. Li, T.A. Barbari, Performance of poly (vinyl alcohol) thin-gel composite ultrafiltration membranes, J. Membr. Sci. 105 (1995) 71-78.

[6] A.G. Fane, C.J.D. Fell, A.G. Waters, Ultrafiltration of protein solutions through permeable membranes - The effect of adsorption and solution environment, J. Membr. Sci. 16 (1983) 211-224.

[7] A. Nabe, E. Staude, G. Belfort, Surface modification of polysulfone ultrafiltration membranes and fouling by BSA solutions, J. Membr. Sci. 133 (1997) 57-72.

[8] K. Burczak, T. Fujisato, M. Hatada, Y. Ikada, Protein permeation through poly (vinyl alcohol) hydrogel membranes, Biomaterials 15 (1994) 231-238. 